\title{
BMJ Open A cross-sectional mixed methods protocol to describe correlates and explanations for a long duration of untreated psychosis among patients with first episode psychosis in Uganda
}

Emmanuel Kiiza Mwesiga, ${ }^{\oplus}$ Noeline Nakasujja, ${ }^{1}$ Linnet Ongeri, ${ }^{2}$ Aggrey Semeere, ${ }^{3}$ Rachel Loewy, ${ }^{4}$ Susan Meffert ${ }^{4}$

To cite: Mwesiga EK, Nakasujja N, Ongeri L, et al. A cross-sectional mixed methods protocol to describe correlates and explanations for a long duration of untreated psychosis among patients with first episode psychosis in Uganda. BMJ Open 2019;9:e028029. doi:10.1136/ bmjopen-2018-028029

- Prepublication history and additional material for this paper are available online. To view these files, please visit the journal online (http://dx.doi org/10.1136/bmjopen-2018028029).

Received 19 November 2018 Revised 16 April 2019 Accepted 7 June 2019
Check for updates

(C) Author(s) (or their employer(s)) 2019. Re-use permitted under CC BY-NC. No commercial re-use. See rights and permissions. Published by BMJ.

For numbered affiliations see end of article.

Correspondence to Dr Emmanuel Kiiza Mwesiga; emwesiga@chs.mak.ac.ug

\section{ABSTRACT}

Introduction Among patients with psychotic disorders, the 'duration of untreated psychosis' (DUP) is a predictor of key outcomes such as symptom remission and quality of life. In sub-Saharan Africa, DUP is up to five times longer than in high-income countries, with many patients going without antipsychotic medication for 5 years or longer. One contributor to this high DUP may relate to cultural norms that drive use of alternative and complementary therapies (ACTs) as first-line treatment strategies, rather than biomedical care with antipsychotic medicine. We aim to ${ }^{1}$ determine the prevalence and factors associated with DUP and ACT use in Uganda, and ${ }^{2}$ Identify factors that drive patient and family choices to use ACT as a first-line treatment strategy.

Methods and analysis We will leverage on an ongoing cohort study at the national psychiatric and teaching hospital in Uganda. The parent study is an observational cohort design following antipsychotic naïve adults with a first episode of psychosis without substance use, HIV/AIDS or syphilis. The embedded study will use a mixed methods design including quantitative assessment of parent study participants with the Nottingham Onset Schedule-DUP to determine the DUP. Qualitative assessment will focus on patient and caregiver perceptions and use of ACT and its impact on DUP among patients with psychosis using indepth interviews.

Ethics and dissemination The study has received ethical approval from the school of medicine research and ethics committee of the college of health sciences at Makerere University. It has also received institutional support to perform the study from the Infectious Diseases Institute and Butabika hospital. Besides publication of the work in reputable peer-reviewed journals, we hope that this work will lead to evidence-based discussions on the need for early interventions to reduce DUP in Uganda.

\section{INTRODUCTION AND BACKGROUND}

Psychotic disorders are among the leading contributors to adult mental illness and disease burden globally. ${ }^{1-3}$ Psychotic disorders are profoundly disabling ${ }^{4}$-schizophrenia,
Strengths and limitations of this study

- A mixed methods study design combines the strengths of both qualitative and quantitative approaches to understand the given problem.

- The Nottingham Onset Schedule duration of untreated psychosis (DUP) version is a well-validated standardised instrument for assessing for the DUP.

- This study also enrols caregivers who are crucial stakeholders in the assessment and mental healthcare of individuals with psychosis.

- As with any cross-sectional study, this investigation is not designed to determine causality.

the most prevalent psychotic disorder, was assigned the highest disability coefficient of all 235 conditions in the 2013 global burden of disease (GBD) study. ${ }^{5}$ GBD disability weights range from 0 (perfect health) to 1 (death). GBD researchers assigned acute schizophrenia a disability weight of $0.778-$ more than three-quarters of the way towards death. Of note, the high GBD disability coefficient still captures only the disability experienced by the affected individual and not the impact of the illness on their close contacts or community. ${ }^{6}$ This burden of psychotic disorders may be reduced by intervening early with antipsychotic medication especially at the first episode of psychosis (FEP). ${ }^{7}$ There are, however, a disproportionately larger numbers of patients from sub-Saharan Africa (SSA) who are not on psychotropic medications or delay in receiving them. ${ }^{8}$

Duration of untreated psychosis (DUP) describes the period in the course of a psychotic illness between the onset of psychotic symptoms and initiation of antipsychotic medication at the FEP. ${ }^{9}$ This delay, DUP, is crucial to psychosis research due 
to its ability to predict future outcomes. ${ }^{9}{ }^{10}$ Changes in brain structure and functioning develop much earlier before the onset of psychotic symptoms ${ }^{11}$ and antipsychotic medication may slow this dysfunction leading to better outcomes. ${ }^{12}$ Patients from SSA have been reported to have DUP of up to 5 years, compared with 1 year in high-income countries (HICs) ${ }^{13-15}$ Shorter DUP of less than 2 years like that seen in HICs is associated with lower symptom severity, higher cognitive functioning, decreased substance use, less disability, better quality of life and better social functioning. ${ }^{13} 1416-19$ The prevalence and factors associated with long DUP in SSA have not been well described. ${ }^{20} 21$

In SSA, alternative and complementary therapies (ACTs) are commonly utilised in the initial treatment of psychotic disorders. ${ }^{22-24}$ These ACTs encompass multiple health and wellness therapies that have not been part of conventional western medicine. ${ }^{25}$ In Uganda, approximately $37 \%$ of patients with psychosis first seek care from ACTs such as faith healers, spiritual healers and traditional healers. ${ }^{23} 26{ }^{27}$ Instead of using antipsychotics, ACT practitioners typically use herbs, psychotherapy, psychodrama, symbolisms, rituals, spirituality and prayer to treat psychosis. ${ }^{28}$ Often, these therapies are used exclusively during the onset of psychosis, which may delay initiation of treatment, leading to longer DUP and potentially worse outcomes. ${ }^{29}$

There is limited and inconsistent literature on what prolongs DUP in SSA - a research gap that hinders the development of effective interventions to reduce DUP. $^{203031}$ There are regional variations in the choice of first contact for mental disorders including psychosis. ${ }^{32} 33$ In a study in South Africa, for example, they reported that the first encounter was a general hospital ${ }^{30}$ while in Uganda it is reported to be ACT. ${ }^{24}$ There is also limited qualitative literature on the use of ACT and how it drives seeking of care and continued care for patients with psychotic disorders. ${ }^{34}$ Given that families seek ACT for psychosis exclusively before biomedical care, ${ }^{23}{ }^{32}$ we hypothesise that the various factors primarily driven by previous use of ACT may be associated with delays in receiving antipsychotic medicine and therefore longer DUP. We undertake this study with the goal of describing the factors associated with long DUP as well as working collaboratively with ACT providers to improve early access to antipsychotic medications.

\section{Aims and objectives}

We will conduct a mixed methods study design employing both quantitative and qualitative approaches concurrently. We aim to determine the prevalence and factors associated with a long DUP among patients with a FEP at Butabika hospital in Uganda. We also aim to document explanations for prior use of alternative and traditional therapies on DUP among patients with FEP presenting to Butabika hospital in Uganda.

Our conceptual framework will be based on Andersen's behavioural model of health service use ${ }^{35}$ (figure 1).

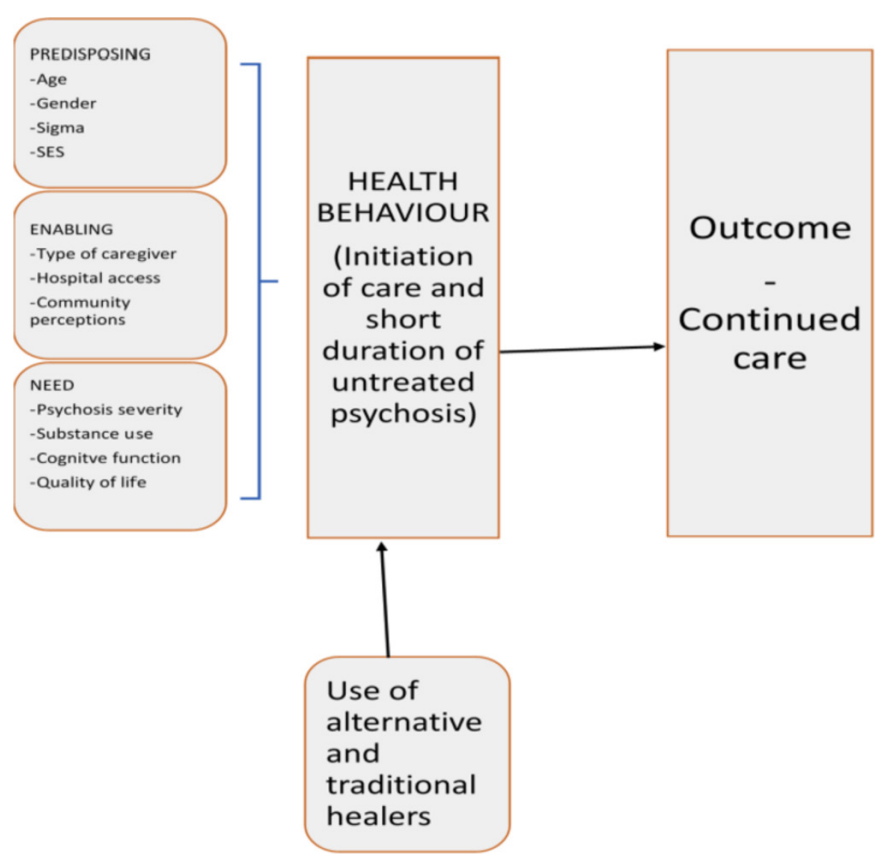

Figure 1 Conceptual framework based on Andersen's behavioural model of health service use showing predisposing enabling and need components.

Briefly, it postulates that predisposing, enabling, and need components in the population predict health behaviour and health outcomes. Predisposing population characteristics may include age, gender, stigma and socioeconomic status. Enabling population characteristics may include type of caregiver (family member vs non-family member), hospital access, availability of care and use of traditional healers. Need population characteristics are the actual or perceived factors that highlight someone needs the services. Need population may include substance use, cognitive function, quality of life and psychosis severity. These factors predict ACT use as a health behaviour which may mediate the DUP defined as the time to initiation of care at Butabika hospital.

\section{METHODS}

\section{Study design and setting}

This mixed methods study will be based at the National Referral and Teaching psychiatric hospital at Butabika (Butabika hospital) (figure 2). Using standardised tools, we will determine the DUP and the factors associated with it while concurrently exploring the role of previous ACT use on DUP.

As a national referral institution, Butabika hospital is an ideal setting for the study of DUP among patients with FEP. As the main tertiary psychiatric institution, it is the final referral point for the whole country and therefore has a wide catchment area of patients from all regions in the country. Hospital records of the year 2018 reviewed in the primary study showed that $67 \%$ of all first contact patients at the hospital have a psychosis diagnosis. This provides an opportunity to gain insights into DUP across 


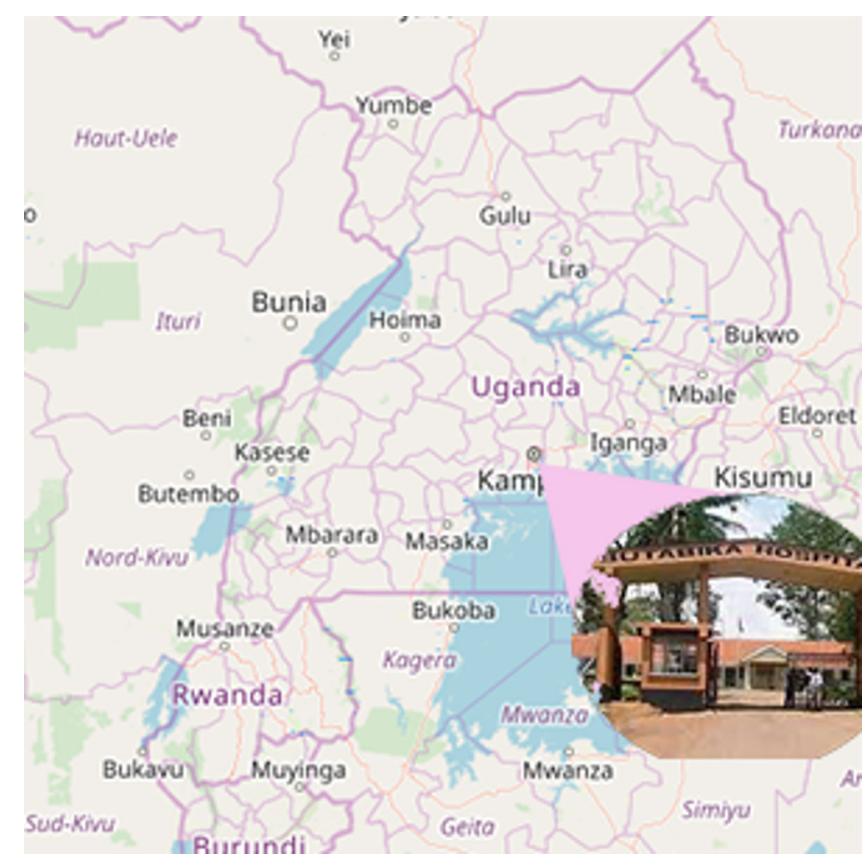

Figure 2 Entrance to Butabika hospital located at Kampala, Uganda.

numerous Ugandan cultures. The hospital serves over 200 psychiatric outpatients per day and has six admitting wards and three convalescent wards that each admit up to 150 patients. In an initial study, we aimed to describe cognitive impairment among patients with a FEP at Butabika hospital.

\section{Participants}

Participants will comprise those who agreed to be contacted for further studies as well as their caregivers. In the primary study, we recruited all patients presenting to Butabika hospital for the first time with a psychotic disorder. Among these participants, we then enrolled inpatients aged 18-60 years with a FEP without prior use of antipsychotic medication or less than 6 weeks of antipsychotic medication. In our original study, we used up to 6 weeks of continuous use of antipsychotic treatment to define the end of DUP. ${ }^{36}$ This was based on literature that highlighted that remission occurred earlier in low-income and middle-income countries. ${ }^{203738}$

Psychosis was confirmed using the Mini International Neuropsychiatric Inventory (MINI). Participants with HIV/AIDS and syphilis were excluded by serological testing. Substance-induced psychotic disorders were also excluded using the MINI. We collected information on sociodemographic characteristics, substance use history, quality of life, psychosis severity, previous childhood trauma and cognitive functioning. Cognitive functioning was assessed when the psychotic symptoms resolved. Participants who, in the original study, reported previous use of ACT were recorded as part of the initial research questions in the sociodemographic questionnaire.

All participants during the consent process were asked whether they can be contacted for further studies. All participants who agreed to be contacted for future studies will be our current study's target population. For the quantitative part of the study, by convenient sampling we will call back all participants who agreed to be contacted for future studies. For the qualitative part of the study, however, we will only purposefully sample a subsection of participants who reported previous use of ACT.

By the end of February 2019, the primary study had enrolled 173 participants. Approximately 30\% of the enrolled participants have reported previous use of ACT. Only one participant had declined to be contacted for future studies.

\section{Consent}

Participants who were previously enrolled in the primary study will be invited back together with a caregiver and asked further questions to guide this new study. Presenting to the hospital following the phone contact will not be taken as consent to participate in the study. Prospective participants will be invited to meet with study personnel in a private room for additional information on the study. A participant information sheet with the study details described will be provided in both Luganda and English (online appendix 1).

The consent process will be carried out by a study psychiatric nurse. The patient will be allowed to read the information sheet and after to ask as many questions concerning the study before consenting to the study. The nurse will emphasise that enrolment is voluntary, and consent can be withdrawn at any stage. The nurse will inquire if the participant needs time to consider accepting to enter the study and if the patient asks for more time enrolment will be scheduled for another day. Before signing the consent form, the study nurse will ask prospective participants to repeat key points they have understood about the study in their own words. If they cannot accurately repeat at least three points, they will be declared unable to consent. A written informed consent will be signed after detailed explanation of the study purpose and thumb prints used in the event of difficulties in writing.

A similar consent process will be carried out for the caregivers who accompany the participants. Caregivers are the people who were listed as the next of kin in the initial study. As the initial study collected up to three next of kin, it will be up to the patient to decide who to come with as next of kin. This will be the individual that we shall consent to take part in the study. All consents and study documents will be in both English and Luganda translations. The participant and the caregiver will be given individual copies of the consent form to keep and a duplicate copy will be kept as a study record by the principal investigator. Consent forms will be stored under lock and key for 3 years as per ethical guidelines. Participants and caregivers who complete the consent process will then undergo eligibility screening. 


\section{Inclusion and exclusion criteria}

The inclusion criteria for the quantitative component of this study, therefore, are as follows: prior acceptance to be contacted for future studies and completion of initial data collection. We will exclude participants who are unable to provide informed consent. For the qualitative arm of the study, we will include participants who previously reported using ACT and have a caregiver willing to participate in the in-depth interviews. We will exclude participants who decline to have the interview audio-recorded.

\section{Bias}

Non-response bias

Participants from far off districts may not be willing to travel for the interviews. We will, however, inform them that they will be reimbursed for their time and transport.

\section{Recall bias}

Assessments for DUP by design are retrospective and thus may be prone to recall bias. It is not unusual to find participants unable to clearly define the onset of the psychotic episode due to the severity of the psychosis or cognitive impairment at psychosis onset which improves as the episode resolves. ${ }^{39}$ As is required when using the Nottingham Onset Schedule-DUP (NOS-DUP), we will invite a close family member to corroborate the events surrounding the onset of the psychosis as is recommended when assessing DUP for patients with psychosis. ${ }^{9}$ There are also some participants who when contacted may have a relapse of their psychotic illness and therefore will not be able to respond to the study questions. ${ }^{32} 33$

\section{Selection bias}

The fact that our original study is based on inpatients with a FEP implies that our sample may be prone to selection bias. However, the rates of previous ACT use in our sample are in keeping with previous literature in the same population. ${ }^{40}$ Also, our objective may not primarily be a pathway to care but more specifically the effect of previous ACT use on initiating care and continuation with care.

\section{Sample size}

For the quantitative arm of the study, we used the KishLeslie formula ${ }^{41}$ to estimate a sample size of 154 participants based on a $95 \%$ confidence limit, $50 \%$ hypothesised percentage of the outcome-initiation of care and an anticipated effect size of $0.4{ }^{42}$ We used a hypothesised percentage of $50 \%$ given the limited literature on how many participants with FEP who initially utilise ACT go on to get care from hospitals. Allowing for a $20 \%$ non-response, we will recruit a minimum of 180 participants.

For the qualitative part of the study, we used a tool designed by Fugard and Potts to estimate sample sizes for qualitative data. ${ }^{43}$ We estimated the most prevalent theme of previous use of ACT of $40 \%$ from previous studies. ${ }^{40} \mathrm{We}$ also anticipated that we will get 10 theme instances. We will therefore perform 30 in-depth interviews. In all, 15 in-depth interviews will involve patients and 15 in-depth interviews will involve caregivers of the selected participants. We estimate that this sample size will be enough to help us reach saturation.

\section{Data collection}

A summary of tools used in the study are listed in table 1. Interview questions will be loaded onto Open Data Kit V.2 (ODK2) using JavaScript software-an established data gathering tool, allowing for offline data entry with later upload to a secure online data server.

The sample for the quantitative component of the study will comprise a convenient sample from all formerly enrolled participants who agreed to be contacted for future studies. The study nurse will approach prospective participants to invite them to learn about the study and rule out a relapse of a psychotic episode. After signing the consent form, the study nurse will commence interviewing the participant using the standardised questionnaires of the NOS-DUP and Barriers to Accessing Care Evaluation (BACE).

For the qualitative component of the study, an interview guide will be developed and piloted that takes into consideration the contextual characteristics, individual characteristics, health behaviours and outcomes like quality of life according to our conceptual framework. Specifically, we will attempt to get an understanding on who made the decision to seek care from ACT initially as well as experiences and challenges while receiving ACT. We will also get a clearer understanding on experiences and challenges while using antipsychotic medication and preferred choices of treatment going forward.

We will purposefully sample from participants who reported previous use of ACT. In the original study, previous ACT use was described as use of a traditional healer/herbalist, use of a spiritual healer (like priests, pastors, sheiks) or use of traditional witch doctor. Among all the participants who reported previous ACT use, we will select five participants and five caregivers from each group to make our sample size. These will then be approached by the study nurse to obtain informed consent. We will then perform in-depth interviews using a standard guide. The interview for both patients and caregivers will entail face-to-face in-depth interviews. The interviews will be done individually with the patient alone and later the caregiver alone. The interviews will be held in a private research room provided by the hospital for the research study. The interview guide was developed by the research team based on input from literature 2329 and preliminary results from the original study. The interview guide was piloted to ensure it captures all the required themes. The qualitative interview will be carried out by a clinical psychologist with previous expertise in carrying out qualitative studies among patients with severe mental illness.

\section{Data analysis plan}

For the quantitative analysis, the length of the DUP will be our dependent variable. A long DUP will be defined 
Table 1 List of tools used in the study

Tool name: variable(s) measured

Sociodemographic questionnaire: age, gender, minimum education attained, household income and employment status

Nottingham Onset Schedule-DUP version (NOS-DUP) $^{48}$ : DUP

\title{
Brief notes
}

Using mapping abilities in Open Data Kit, we will be able to collect data on names of health facilities closest to the participants home to calculate distances to available services in the area.

Short, guided interview for recording several time points in the onset of psychosis. Onset is conceptualised as comprising a prodrome, emergence of positive psychotic symptoms and build-up of symptoms leading to a definite diagnosis (transition into psychosis). The NOSDUP has been shown to have good validity in describing previous psychotic experiences with an intraclass correlation of 0.89 in some studies. ${ }^{48}$

\author{
Barriers to Accessing Care Evaluation \\ (BACE) $^{49}$ : barriers to accessing care
}

The BACE measures barriers to accessing medical care including antipsychotic treatment. It asks about 30 different barriers to care and has a special focus on stigma-related barriers. It has a 'treatment stigma' subscale to assess to what extent the stigma associated with mental healthcare has been a barrier for an individual. It has good psychometric properties and is also being used in studies in LMICs. ${ }^{50} 51$

Child Trauma Questionnaire (CTQ) ${ }^{55 *}$ : provides a brief method for screening of childhood traumatic experiences

The MINI International Neuropsychiatric Interview $^{57 \star}$ : psychosis diagnoses

The CTQ is as 28 -item questionnaire that takes $10-15$ min to administer. It also includes a minimisation/denial scale for detecting individuals who may be under-reporting traumatic events. It has been used before in sub-Saharan Africa. ${ }^{56}$

The MINI 7.0.2 is a semistructured interview guide for making Diagnostic and Statistical Manual of Mental Disorders, fifth edition (DSM-5) diagnoses. We administer module A (depressive disorder), module C (bipolar disorder), module I (alcohol use), module J (other substances) and module $\mathrm{K}$ (psychotic disorders).

\section{Alcohol Smoking and Substance Use} Involvement Test (ASSIST) ${ }^{58 *}$ : severity of substance use in the 3 months prior to enrolment
The ASSIST was developed for the WHO to detect and manage substance use and related problems in primary and general medical care settings. The ASSIST specifically collects information on nine major classes of substances that include tobacco, cannabis, alcohol, cocaine, amphetamines, inhalants, sedatives, hallucinogens and opioids. It also has a 10th category that collects any substance that might not fall in the nine general classes. The ASSIST is able to categorise different substance involvement into low, moderate and high risk. It has been previously used in our setting and in LMICs the reliability ranges from 0.81 to $0.95 .^{59}$

Positive and Negative Signs and Symptoms
of Schizophrenia (PANSS)
severity

\section{WHO Quality of Life tool-Brief version} (WHOQOL-BREF) $^{61 *}$ : quality of life in four domains namely physical health, psychological health, social relationships and environment

Neuroscreen $^{64 *}$ :
The PANSS is a 30 -item clinician rated scale. It is used to determine resolution of positive psychotic symptoms before the assessment of cognitive function. Participants had to score less than 3 out of 7 in all domains to determine psychosis resolution.

Comprises 26 items. It is a shorter version of the original instrument that may be more convenient for use in large research studies or clinical trials. It has been previously used and validated in our setting. ${ }^{62} 63$ It can be administered in approximately $10 \mathrm{~min}$. In field trials, it was found to be reliable with Cronbach scores of $0.7 .^{63}$

It is a collection of brief psychological screening tests administered via a smart phone application. It assesses six cognitive domains that include learning and memory, working memory, processing speed, motor speed and executive function. It has been validated for use in low- and middle-income settings. The tests in the battery are described elsewhere. ${ }^{65}$ In specific populations, it has been found to have a sensitivity of $88.9 \%$ and sensitivity of $62.5 \%$. The validity in first episode of psychosis will be described by the primary study.

*Data were previously collected by primary study and provides independent variables in assessing the association between previous alternative and complementary therapy use and DUP.

DUP, duration of untreated psychosis; LMIC, low- and middle-income countries.

as more than 2years before receiving antipsychotic medication after onset of psychotic symptoms. ${ }^{9} 4546$ We will use multivariate linear regression analyses to determine the association between ACT use with DUP while controlling for age and gender. For the qualitative objective, grounded theory approach will be used. ${ }^{47}$ The goal of grounded theory approach is a theoretical analysis of how people resolve a major concern. A system of openended data evaluation that allows theory to merge from the dataset will guide data analysis. Interview material will be recorded in audio files, and then transcribed by the clinical psychologist. Coding will be done by the PI and the clinical psychologist to ensure we get similar themes. Data analysis will be done using Atlas software.

\section{Data quality control}

In using standardised tools like the NOS-DUP version ${ }^{48}$ and the BACE, ${ }^{49}$ the authors hope to limit this bias. The NOS-DUP has been shown to have good validity in describing previous psychotic experiences with an intraclass correlation of 0.89 in some studies. ${ }^{48}$ BACE has good psychometric properties and is also being used before in studies in low-income and middle-income countries. 5051

Both the NOS-DUP and the BACE will be translated from English to Luganda which is the the most common local language by two independent individuals from the local community with the goal of producing a translation that is appropriate for the lowest level of location education. The two translations will then be compared, 
and discrepancies resolved. Then, two additional individuals from the local community will back-translate from Luganda to English. Versions will be compared, and discrepancies will be resolved. After these translation procedures, the instrument will be pilot tested with at least five individuals' representative of the local respondent education level. Translations will be adjusted based on pilot participant understanding and feedback until the instrument can be read word-for-word and clearly understood by responders without any additional clarification. ${ }^{52}$ The quality of the study will be further ensured through adequate training of interviewers (psychiatric clinical officer, medical officer and clinical psychologist), pre-testing and piloting the tools before use. ODK also allows for validation of data as it is being collected to prevent errors during data entry.

\section{Patient and public involvement}

The patients and public were involved indirectly in this study by agreeing to provide information for the primary study. Preliminary analyses from the primary study that showed a large percentage of patients use ACT informed the need for the current study. Information of this study will highlight if ACT use delays initiation of care and its overall effects on patient outcomes like cognitive function. This information will be shared with the administration of Butabika hospital who by law set the mental health policy agenda for the country.

\section{Discussion}

Any intervention that seeks to improve outcomes in patients with psychosis needs to ensure that there is a short DUP. ${ }^{21} 5354$ Early, correct and sustained treatment with antipsychotic medication is essential to improved quality of life, recovery, cognitive and social functioning. ${ }^{7}$ In Uganda, there is limited literature on the DUP and the factors associated. Also, previous literature has highlighted the use of ACT as an initial treatment choice for patients with psychosis. ${ }^{24}{ }^{29}$ It is essential to gain an understanding on the role of ACT on DUP. This can ensure that interventions are designed to ensure there is a reduction in DUP to ensure improved outcomes. Therefore, assessing for DUP and associated factors as well as qualitative research on the influence of ACT on DUP and care-seeking behaviour will be central to the proposed research.

\section{Ethics and dissemination}

Ethical approval has been received from ethics committees at the school of medicine research and ethics committee of the college of health sciences at Makerere University (\#REC REF 2017-153) as well as the Uganda National Council of Science and Technology (HS237ES). All recruitment, consent and data collection assessments will be done in English or Luganda which is commonly spoken in the region. All recruitment and enrolment will be done after the normal routine care so as not to interfere with the clinic routine. Participants will be reimbursed $\$ 6$ (approximately sh20 000) for their time, either on completion of the full study assessment or when consent is withdrawn. Caregivers who participate in the qualitative study will also be paid $\$ 6$ (approximately sh20 $000)$ separately from the patients they represent. No individual participant data will be reported in the published article. Participants found to be undergoing treatment seeking stigma will be referred to social workers and clinical psychologists for psycho education. We will also refer participants found to have a relapse of the psychotic illness to the clinic for management.

The results from this work will be shared with the management of Butabika hospital who are responsible for determining mental health policy in Uganda. We hope that this work will lead to evidence-based discussions on the need for early interventions to reduce DUP in Uganda. We will also publish our results in peer-reviewed journals. Our results will also be shared widely with the scientific world at conferences as oral or poster presentations.

\section{Article summary}

This is a research protocol of a mixed methods study that aims to determine the association between previous ACT use with DUP among patients with a FEP in Uganda.

\section{Author affiliations}

${ }^{1}$ Department of Psychiatry, College of Health Sciences, Makerere University, Kampala, Uganda

${ }^{2}$ Center for Clinical Research, Kenya Medical Research Institute, Nairobi, Kenya ${ }^{3}$ Infectious Diseases Institute, College of Health Sciences, Makerere University, Kampala, Uganda

${ }^{4}$ Department of Psychiatry, University of California San Francisco, San Francisco, California, USA

Acknowledgements We are grateful to the patients who accepted to be contacted for further studies. We also acknowledge the Infectious Diseases Institute (IDI) who through their systems ensure that the study will be carried out according to ethical guidelines. IDI reviewed the manuscript to ensure there was no plagiarism and that the manuscript was written according to the BMJ Open guidelines.

Contributors EKM conceptualised the research idea. EKM, RL, NN, LO, AS and SM were responsible for determining the content and scope of the study. EKM, LO, AS and SM were responsible for determining the design and methods of the study. $\mathrm{NN}$ and RL were responsible for determining the study tools and were content experts. All authors were also involved in writing this protocol and accepted the final version.

Funding The corresponding author was awarded the prestigious Fogarty fellowship (2018 to 2019) to be able to perform this study. This project is supported by the Fogarty International Centre (FIC) and the National Institutes of Mental Health of the National Institute of Health (NIH) under award number D43TW009343 and the University of California Global Health Institute (UCGHI).

Map disclaimer The depiction of boundaries on the map(s) in this article do not imply the expression of any opinion whatsoever on the part of BMJ (or any member of its group) concerning the legal status of any country, territory, jurisdiction or area or of its authorities. The map(s) are provided without any warranty of any kind, either express or implied.

Disclaimer The consent is solely the responsibility of the authors and does not necessarily represent the official views of the NHI or UCGHI.

Competing interests Dr. Rachel Loewy serves as faculty of the Lundbeck International Neuroscience Foundation.

Patient consent for publication Not required.

Provenance and peer review Not commissioned; externally peer reviewed. 
Open access This is an open access article distributed in accordance with the Creative Commons Attribution Non Commercial (CC BY-NC 4.0) license, which permits others to distribute, remix, adapt, build upon this work non-commercially, and license their derivative works on different terms, provided the original work is properly cited, appropriate credit is given, any changes made indicated, and the use is non-commercial. See: http://creativecommons.org/licenses/by-nc/4.0/.

\section{REFERENCES}

1. Ruggeri M, Leese M, Thornicroft $\mathrm{G}$, et al. Definition and prevalence of severe and persistent mental illness. Br J Psychiatry 2000;177:149-55.

2. Hyman S, Parikh R, Collins PY, et al. Adult Mental Disorders.

3. Association AP. DSM 5: American Psychiatric Association. 2013.

4. Rössler W, Salize HJ, van Os J, et al. Size of burden of schizophrenia and psychotic disorders. Eur Neuropsychopharmacol 2005:15:399-409.

5. Salomon JA, Vos T, Hogan DR, et al. Common values in assessing health outcomes from disease and injury: disability weights measurement study for the Global Burden of Disease Study 2010. Lancet 2012;380:2129-43.

6. Awad AG, Voruganti LN. The burden of schizophrenia on caregivers: a review. Pharmacoeconomics 2008;26:149-62.

7. Marshall M, Rathbone J. Early intervention for psychosis. Cochrane Database Syst Rev 2011;15.

8. Read U. "I want the one that will heal me completely so it won't come back again": the limits of antipsychotic medication in rural Ghana. Transcult Psychiatry 2012;49(3-4):438-60.

9. Souaiby L, Gaillard R, Krebs MO. [Duration of untreated psychosis: a state-of-the-art review and critical analysis]. Encephale 2016;42:361-6.

10. Santesteban-Echarri O, Paino M, Rice S, et al. Predictors of functional recovery in first-episode psychosis: A systematic review and meta-analysis of longitudinal studies. Clin Psychol Rev 2017;58:59-75.

11. Kahn RS, Sommer IE. The neurobiology and treatment of firstepisode schizophrenia. Mol Psychiatry 2015;20:84-97.

12. Puri BK. Brain tissue changes and antipsychotic medication. Expert Rev Neurother 2011;11:943-6.

13. Chiliza B. Early intervention in schizophrenia in developing countries: focus on duration of untreated psychosis and remission as a treatment goal. (1369-1627 (Electronic)).

14. Farooq $S$, Waheed $\mathrm{W}$. The relationship between the duration of untreated psychosis and outcome in low-and-middle income countries: a systematic review and meta analysis. (0920-9964 (Print).

15. Kane JM, Robinson DG, Schooler NR, et al. Comprehensive versus usual community care for first-episode psychosis: 2-year outcomes from the NIMH RAISE early treatment program. Am J Psychiatry 2016;173:362-72.

16. Marshall M, Rathbone J. Early intervention for psychosis. (1469-493X (Electronic)).

17. Murru A, Carpiniello B. Duration of untreated illness as a key to early intervention in schizophrenia: a review. Neurosci Lett 2016;4:30745-5.

18. Albert $\mathrm{N}$, Melau $\mathrm{M}$, Jensen $\mathrm{H}$, et al. The effect of duration of untreated psychosis and treatment delay on the outcomes of prolonged early intervention in psychotic disorders. NPJ Schizophr 2017;3:017-34.

19. Perkins DO, Gu H, Boteva K, et al. Relationship between duration of untreated psychosis and outcome in first-episode schizophrenia: a critical review and meta-analysis. Am J Psychiatry 2005;162:1785-804.

20. Chiliza B, Asmal L, Emsley R. Early intervention in schizophrenia in developing countries: focus on duration of untreated psychosis and remission as a treatment goal. Int Rev Psychiatry 2012;24:483-8.

21. Emsley R, Chiliza B, Schoeman R. Predictors of long-term outcome in schizophrenia. Curr Opin Psychiatry 2008;21:173-7.

22. Sorketti EA, Zainal NZ, Habil MH. The characteristics of people with mental illness who are under treatment in traditional healer centres in Sudan. Int J Soc Psychiatry 2012;58:204-16.

23. Abbo C, Ekblad S, Waako P, et al. The prevalence and severity of mental illnesses handled by traditional healers in two districts in Uganda. Afr Health Sci 2009;1:S16-22.

24. Abbo C, Okello ES, Musisi S, et al. Naturalistic outcome of treatment of psychosis by traditional healers in Jinja and Iganga districts, Eastern Uganda - a 3- and 6 months follow up. Int J Ment Health Syst 2012;6:13-4458.
25. Woolhouse M. Complementary therapies in mental health care. Aust Fam Physician 2007;36:247.

26. Paruk S, Jhazbhay K, Singh K, et al. Clinical correlates of first episode early onset psychosis in KwaZulu-Natal, South Africa. J Child Adolesc Ment Health 2015;27:103-11.

27. Abbo C, Okello ES, Musisi S, et al. Naturalistic outcome of treatment of psychosis by traditional healers in Jinja and Iganga districts, Eastern Uganda - a 3- and 6 months follow up. Int J Ment Health Syst 2012;6:13

28. Abbo $\mathrm{C}$, Odokonyero $\mathrm{R}$, Ovuga $\mathrm{E}$. A narrative analysis of the link between modern medicine and traditional medicine in Africa: a case of mental health in Uganda. Brain Res Bull 2019;145.

29. Abbo C. Profiles and outcome of traditional healing practices for severe mental illnesses in two districts of Eastern Uganda. Glob Health Action 2011;4:7117.

30. Tomita A, Burns JK, King $\mathrm{H}$, et al. Duration of untreated psychosis and the pathway to care in KwaZulu-Natal, South Africa. J Nerv Ment Dis 2015;203:222-5.

31. Oosthuizen P, Emsley RA, Keyter N, et al. Duration of untreated psychosis and outcome in first-episode psychosis. Perspective from a developing country. Acta Psychiatr Scand 2005;111:214-9.

32. Burns JK, Tomita A. Traditional and religious healers in the pathway to care for people with mental disorders in Africa: a systematic review and meta-analysis. Soc Psychiatry Psychiatr Epidemiol 2015;50:867-77.

33. Chidarikire S, Cross M, Skinner I, et al. Treatments for people living with schizophrenia in Sub-Saharan Africa: an adapted realist review. Int Nurs Rev 2018;65:78-92.

34. Zuma T, Wight D, Rochat T, et al. The role of traditional health practitioners in Rural KwaZulu-Natal, South Africa: generic or mode specific? BMC Complement Altern Med 2016;16:304

35. Gelberg L, Andersen RM, Leake BD. The Behavioral Model for Vulnerable Populations: application to medical care use and outcomes for homeless people. Health Serv Res 2000;34:1273-302.

36. Breitborde NJ, Srihari VH, Woods SW. Review of the operational definition for first-episode psychosis. Early Interv Psychiatry 2009;3:259-65.

37. Emsley R. Remission in first-episode psychosis: predictor variables and symptom improvement patterns. (1555-2101 (Electronic)).

38. Rangaswamy $T$, Mangala R, Mohan G, et al. Early intervention for first-episode psychosis in India. East Asian Arch Psychiatry 2012;22:94-9.

39. Sheffield JM, Karcher NR, Barch DM. Cognitive deficits in psychotic disorders: a lifespan perspective. Neuropsychol Rev 2018;28:509-33.

40. Abbo C, Ekblad S, Waako P, et al. The prevalence and severity of mental illnesses handled by traditional healers in two districts in Uganda. Afr Health Sci 2009;9(Suppl 1):S16-22.

41. Kish L, Frankel MR. Inference from Complex Samples. Journal of the Royal Statistical Society: Series B 1974;36:1-22.

42. Davis GP, Tomita A, Baumgartner JN, et al. Substance use and duration of untreated psychosis in KwaZulu-Natal, South Africa. S Afr J Psychiatr 2016;22:7.

43. Fugard AJB, Potts HWW. Supporting thinking on sample sizes for thematic analyses: a quantitative tool. Int J Soc Res Methodol 2015;18:669-84.

44. Abbo C, Ekblad S, Waako P, et al. Psychological distress and associated factors among the attendees of traditional healing practices in Jinja and Iganga districts, Eastern Uganda: a crosssectional study. Int J Ment Health Syst 2008;2:16-4458.

45. Savill M, Gosdin M, Patel P, et al. How do early psychosis services define and operationalize the duration of untreated psychosis? J Behav Health Serv Res 2019;46:497-508.

46. Murru A, Carpiniello B. Duration of untreated illness as a key to early intervention in schizophrenia: a review. Neurosci Lett 2018;669:59-67.

47. Charmaz K, Belgrave LL. Grounded theory. The Blackwell encyclopedia of sociology. 2007.

48. Singh SP, Cooper JE, Fisher HL, et al. Determining the chronology and components of psychosis onset: the Nottingham Onset Schedule (NOS). Schizophr Res 2005;80:117-30.

49. Clement S, Brohan E, Jeffery D, et al. Development and psychometric properties the Barriers to Access to Care Evaluation scale (BACE) related to people with mental ill health. BMC Psychiatry 2012;12:36

50. Silva L, Silva PF, Gadelha A, et al. Adaptation of the Barriers to Access to Care Evaluation (BACE) scale to the Brazilian social and cultural context. Trends Psychiatry Psychother 2013;35:287-91.

51. Luitel NP, Jordans MJD, Kohrt BA, et al. Treatment gap and barriers for mental health care: a cross-sectional community survey in $\mathrm{Nepal}$ PLoS One 2017;12:e0183223. 
52. van Ommeren M, Sharma B, Thapa S, et al. Preparing Instruments for transcultural research: use of the translation monitoring form with nepali-speaking bhutanese refugees. Transcult Psychiatry 1999;36:285-301.

53. Perez J, Russo DA, Stochl J, et al. Understanding causes of and developing effective interventions for schizophrenia and other psychoses.

54. Farooq S, Large M, Nielssen $\mathrm{O}$, et al. The relationship between the duration of untreated psychosis and outcome in low-and-middle income countries: a systematic review and meta analysis. Schizophr Res 2009;109(1-3):15-23.

55. Bernstein DP. Initial reliability and validity of a new retrospective measure of child abuse and neglect. (0002-953X (Print)).

56. Kilian S, Asmal L, Chiliza B, et al. Childhood adversity and cognitive function in schizophrenia spectrum disorders and healthy controls: evidence for an association between neglect and social cognition. Psychol Med 2018;48:2186-93.

57. Sheehan D, Janavs J, Sheehan K, et al. Mini international neuropsychiatric interview 6.0: high prevalence disorders, english version. Tampa, FL: University of South Florida, 2010.

58. WHO ASSIST Working Group. The Alcohol, Smoking and Substance Involvement Screening Test (ASSIST): development, reliability and feasibility. Addiction 2002;97:1183-94.
59. van der Westhuizen $\mathrm{C}$, Wyatt G, Williams JK, et al. Validation of the alcohol, smoking and substance involvement screening test in a lowand middle-income country cross-sectional emergency centre study. Drug Alcohol Rev 2016;35:702-9.

60. Kay SR, Fiszbein A, Opler LA. The positive and negative syndrome scale (PANSS) for schizophrenia. Schizophr Bull 1987;13:261-76.

61. Skevington SM, Lotfy M, O'Connell KA. The World Health Organization's WHOQOL-BREF quality of life assessment: psychometric properties and results of the international field trial. A report from the WHOQOL Group. Quality of Life Research 2004;13:299-310.

62. Mwesiga EK, Mugenyi L, Nakasujja N, et al. Depression with pain co morbidity effect on quality of life among HIV positive patients in Uganda: a cross sectional study. Health Qual Life Outcomes 2015;13:206.

63. Skevington SM, Lotfy M, O'Connell KA. The World Health Organization's WHOQOL-BREF quality of life assessment: psychometric properties and results of the international field trial. A report from the WHOQOL group. Qual Life Res 2004;13:299-310.

64. Robbins RN, Brown $\mathrm{H}$, Ehlers A, et al. A smartphone app to screen for HIV-Related neurocognitive impairment. (1839-7808 (Linking)).

65. Robbins RN, Brown H, Ehlers A, et al. A smartphone app to screen for HIV-related neurocognitive impairment. J Mob Technol Med 2014;3:23-36. 